\title{
Serum butyrylcholinesterase activities in patients with non-alcoholic fatty liver disease. Comparison with liver proteosynthetic function and liver fibrosis
}

\author{
Turecky $\mathrm{L}^{1}$, Kupcova $\mathrm{V}^{2}$, Durfinova $\mathrm{M}^{1}$, Uhlikova $\mathrm{E}^{1}$ \\ Institute of Medical Chemistry, Biochemistry and Clinical Biochemistry, Medical School, Comenius University, \\ Bratislava, Slovakia. ladislav.turecky@fmed.uniba.sk
}

\begin{abstract}
BACKGROUND: We aimed to determine how does butyrylcholinesterase (BChE) activity change in the serum of NAFLD patients, whether there was a relationship between BChE and the severity of NAFLD and whether $\mathrm{BChE}$ could be used to distinguish the patients with simple hepatic steatosis from the patients with nonalcoholic steatohepatitis.

METHODS: The study group consisted of 64 patients with NAFLD. Patients were examined for fatty liver index and in the serum, we investigated BChE activities and the concentrations of prealbumin, cholesterol, HDL-cholesterol, triacylglycerols and hyaluronic acid (HA). We also used FIB-4 index to evaluate liver fibrosis. RESULTS: BChE activity was significantly increased in NAFLD patients compared to the controls (4711 U/I vs $4028 \mathrm{U} / \mathrm{l})$. Patients with higher concentrations of serum triacylglycerols and non-HDL cholesterol had also significantly higher activities of BChE. The comparison of BChE activity and parameters of liver fibrosis (HA and FIB-4) showed a significant negative correlation between these parameters. Patients with an increased concentration of HA had significantly lower BChE than the controls (3111 U/l vs $4028 \mathrm{U} / \mathrm{l})$.

CONCLUSIONS: Our results showed increased BChE values in NAFLD patients. The comparison of changes in $\mathrm{BChE}$ activity with the changes in prealbumin levels and changes of both fibro markers showed that the examination of BChE activity could help to differentiate NAFLD patients with a simple hepatic steatosis from those with an advanced disease (Tab. 1, Fig. 7, Ref. 28). Text in PDF www.elis.sk KEY WORDS: butyrylcholinesterase, non-alcoholic fatty liver disease, hyaluronic acid, FIB-4, fatty liver index.
\end{abstract}

\section{Introduction}

Non-alcoholic fatty liver disease (NAFLD) is a well-distinguished clinical entity affecting people worldwide. It is characterized by the accumulation of triacylglycerols in the liver. The deposition of triacylglycerols in hepatocytes in the form of fat droplets, which occurs during hepatic steatosis, may be a condition that facilitates the development of other pathological processes such as the inflammatory reaction and necrosis, which may result in fibrosis, resp. liver cirrhosis. An excessive oxidation of fatty acids can result in oxidative stress with free radical formation and in mitochondrial dysfunction, a condition known as lipotoxicity. The immune response to the lipotoxic effect involves inflammatory and regenerative processes that may potentiate fibrogenesis

${ }^{1}$ Institute of Medical Chemistry, Biochemistry and Clinical Biochemistry, Medical School, Comenius University, Bratislava, Slovakia, and ${ }^{2} I I I r d$ Department of Medicine, Medical School, Comenius University, Bratislava, Slovakia

Address for correspondence: $\mathrm{L}$. Turecky, MD, $\mathrm{PhD}$, Institute of Medical Chemistry, Biochemistry and Clinical Biochemistry, Medical School, Comenius university, Sasinkova 2, SK-811 08 Bratislava, Slovakia.

Acknowledgement: This work was supported by The Independent research grants of the Ministry of Education, Science, Research and Sport of the Slovak Republic VEGA (grant number 1/0826/18). and the progression of NAFLD to non-alcoholic steatohepatitis (NASH) or even to liver cirrhosis. The severity of this disorder is that up to $10-20 \%$ of the individuals with NAFLD progress to NASH or liver cirrhosis, eventually to hepatocellular carcinoma $(1,2)$. While simple liver steatosis has a relatively benign prognosis, NASH is characterized by a high risk of progression to liver cirrhosis and hepatocellular carcinoma. Currently, NAFLD is the most common cause of chronic hepatopathies. Epidemiological studies showed that there has been a significant increase in the prevalence of NAFLD in many countries recently $(3,4)$.

Serum cholinesterase (EC 3.1.1.8, pseudocholinesterase, $\mathrm{BChE}$ ) is a secretory enzyme synthesized in the liver and secreted into the blood. It is not a single enzyme, but a group of several isoenzymes. The circulating enzyme is a tetramer composed of 4 identical subunits and with a m.w. of $342 \mathrm{kDa}(5)$. The true physiological function of serum cholinesterase is not yet known. $\mathrm{BChE}$ is able to hydrolytically cleave various hydrophilic and hydrophobic esters of choline (6) and is well known to be involved in the hydrolysis of several xenobiotics such as: succinylcholine, mivacurium, procaine, chloroprocaine, tetracaine, cocaine and heroin (5). Examination of serum cholinesterase activity is used in clinical practice as a parameter to evaluate the proteosynthetic function of the liver. The determination of $\mathrm{BChE}$ activity is also used to monitor liver function after liver transplantation. 
Tab. 1. Values of examined biochemical parameters in patients with NAFLD and healthy controls.

\begin{tabular}{|c|c|c|c|c|c|c|}
\hline & $\begin{array}{c}\text { TAG } \\
\text { mmol.1 }\end{array}$ & $\begin{array}{c}\text { nonHDL-ch } \\
\text { mmol.1 }\end{array}$ & $\begin{array}{l}\text { Total-ch } \\
\text { mmol.1 }\end{array}$ & $\begin{array}{l}\text { BChE } \\
\text { U. } 1^{-1}\end{array}$ & $\begin{array}{l}\text { Prealbumin } \\
\text { mg. } 1^{-1}\end{array}$ & $\begin{array}{l}\text { Hyaluronic acid } \\
\text { ng. } 1^{-1}\end{array}$ \\
\hline Controls & $0.98 \pm 0.12$ & $3.24 \pm 0.14$ & $4.60 \pm 0.14$ & $4028 \pm 221$ & $236 \pm 11$ & $28.5 \pm 1.9$ \\
\hline NAFLD & $1.86 \pm 0.15$ & $3.95 \pm 0.13$ & $5.14 \pm 0.14$ & $4711 \pm 175$ & $236 \pm 9$ & $39.3 \pm 4.8$ \\
\hline Statistical significance & $\mathrm{p}<0.01$ & $\mathrm{p}<0.01$ & $\mathrm{p}<0.05$ & $\mathrm{p}<0.01$ & NS & $\mathrm{p}<0.005$ \\
\hline
\end{tabular}

TAG - triacylglycerols, nonHDL-ch - non-HDL cholesterol, total-ch - total cholesterol, BChE - butyrylcholinesterase, NS - not significant. Results are given as the mean \pm SEM.

Some publications suggest that cholinesterase activity may be increased in the patients with obesity, hyperlipoproteinemia and diabetes $(7,8,9)$.

The aim of our study was to determine how does BChE activity change in the serum of the patients with NAFLD. Whether there is a relationship between $\mathrm{BChE}$ activity and a disorder of lipoprotein metabolism, or the severity of NAFLD, and last, but not least, whether the determination of BChE activity can be used to distinguish the patients with simple hepatic steatosis from the patients with NASH.

\section{Material and methods}

NAFLD patients were enrolled in the hepatologic outpatient clinic at the University Hospital Bratislava-Kramáre. Enrolment criteria were as followed: a sonographically present steatosis and absence of any known or detected acute or chronic liver disease (viral, autoimmune, toxicity).

The study group consisted of 64 patients, 32 men and 32 women. The age of the patients ranged from 23 to 66 years for men, between 29 and 76 years for women, the mean age in the group was 55.2 years, 58.3 years for women and 52.3 years for men. Patients were examined for fatty liver index (FLI) according to the original method of Bedogni et al (10), and the final algorithm was modified on the basis of works of Fedchuk et al (11) and Motamed et al (12), and took into account body mass index, waist circumference, serum triacylglycerol levels, and serum gamma-glutamyltransferase activity. FLI values above 60 indicate the presence of steatosis, with a positive predictive value of this index at values above 60 being $99 \%$ and a negative predictive value of $15 \%$. The FLI value in our group of patients was over 60 in $89 \%$ of the members of the group. None of the patients in our group had FLI value below 30 , which ruled out the presence of liver steatosis.

Regarding the aetiology of NAFLD in our group of patients, in most cases it was a non-alcoholic hepatic steatosis, while in several patients it was not possible to exclude alcohol consumption in the past and the share of alcohol in the pathogenesis of steatotic liver damage. In most cases, obesity, hyperlipoproteinemia, metabolic syndrome and diabetes mellitus played a role in the mechanism of hepatic steatosis.

Patients were examined for the body mass index (BMI), with only $10 \%$ of patients having a normal BMI (values 18.5-24.9 $\mathrm{kg} / \mathrm{m}^{2}$ ), $38 \%$ of patients were overweight according to their BMI (values $25-29.9 \mathrm{~kg} / \mathrm{m}^{2}$ ), $47 \%$ had BMI values corresponding to obesity (values $30-39.9 \mathrm{~kg} / \mathrm{m}^{2}$ ) and $5 \%$ of patients were even extremely obese (BMI values above $40 \mathrm{~kg} / \mathrm{m}^{2}$ ). Diabetes mellitus was present in $58 \%$ of the patients.
The control group consisted of 30 individuals ( 16 females, 14 males) without clinical signs of the disease. These were medical students, medical staff and their families. The average age was 45.1 years (ranging from 22 to 70 years). None of the members of the control group had diabetes mellitus or had elevated serum activities of hepatic enzymes (ALT, AST and GGT).

Blood was collected in the morning, on an empty stomach under standard conditions (after 12 hours of fasting). We collected blood without the addition of an anticoagulant and individual biochemical parameters were examined in blood serum.

We used commercial diagnostic kits to determine serum prealbumin, cholesterol, HDL-cholesterol and triacylglycerols. We investigated the butyrylcholinesterase activity kinetically at $25^{\circ} \mathrm{C}$ using butyryl thiocholine iodide as substrate. The concentration of hyaluronic acid was examined immunochemically by ELISA method, using a commercial diagnostic kit from CORGENIX, Inc., Broomfield, CO, USA. The principle of the method used for the determination of hyaluronic acid is based on a sandwich technique using hyaluronic acid binding protein (HABP) obtained from bovine cartilage. We also used the FIB-4 index to evaluate liver fibrosis. FIB-4 is an index based on the evaluation of platelet counts, serum alanine aminotransferase (ALT) and aspartate aminotransferase (AST) activities, and patient age (13). We calculated it according to the formula FIB-4 = age [years] x AST [U.1 $\left.1^{-1}\right] /$ platelets $\left[10^{9} .1^{-1}\right] \times$ VALT $\left[\right.$ U. $\left.1^{-1}\right]$.

Results are expressed as the mean \pm standard error of mean (SEM). We used non-parametric methods for statistical evaluation of the results. We performed calculations using the statistical program SPSS (Statistical Package for the Social Sciences, SPSS Inc., Chicago, IL, USA) version 19 for Microsoft Windows. A p value of $<0.05$ was taken as significant.

\section{Results}

The values of biochemical parameters examined in the group of patients with non-alcoholic fatty liver disease (NAFLD) are shown in Table 1. Butyrylcholinesterase activity (BChE) was statistically significantly increased in the serum of NAFLD patients compared to the healthy controls. The values of the examined serum lipid parameters were higher in the patients with NAFLD than in the control group. We recorded the most significant increase in the case of triacylglycerols (approximately $90 \%$ ). When cholesterol levels were examined, the increase in non-HDL-cholesterol levels was more pronounced than the increase in total cholesterol. The concentration of hyaluronic acid examined as an indicator of hepatic fibrogenesis was higher in NAFLD patients than in the controls, but its mean value did not exceed the upper limit of the reference 


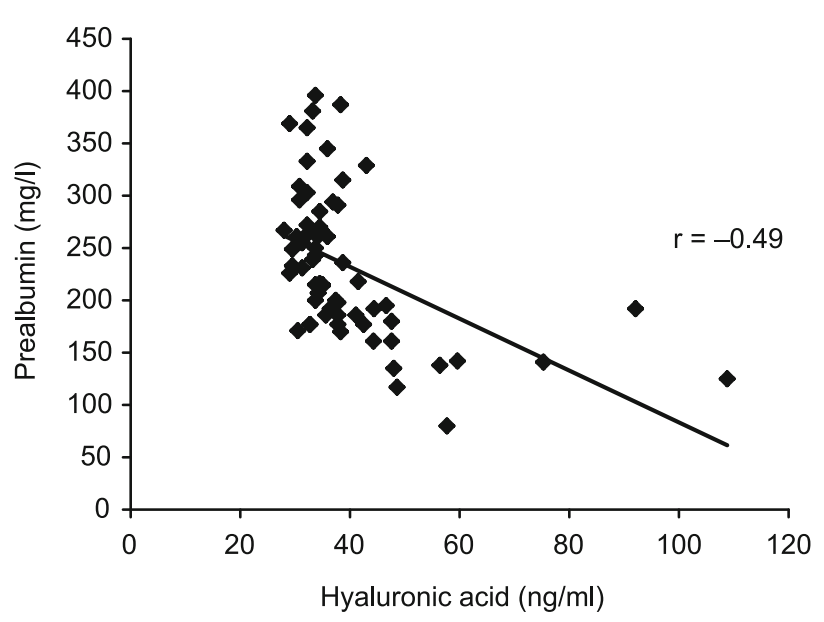

Fig. 1. Correlation between serum prealbumin and hyaluronic acid levels in patients with NAFLD $(p<0.05)$.

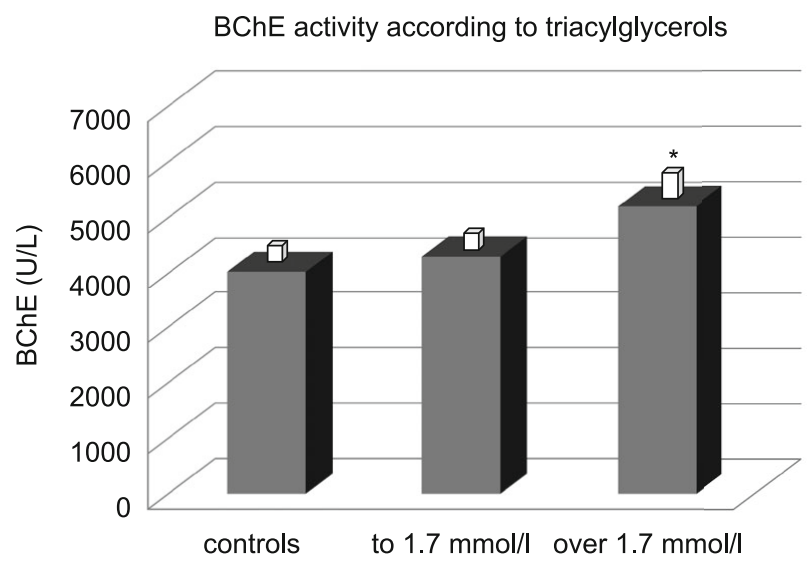

Fig. 2. BChE activity in the serum of patients with NAFLD divided by the level of triacylglycerols. *Statistically significantly higher than in controls and in the group of patients with triacylglycerols less than $1.7 \mathrm{mmol.}^{-\mathrm{l}^{-1}}$.

BChE activity according to non-HDL-cholesterol

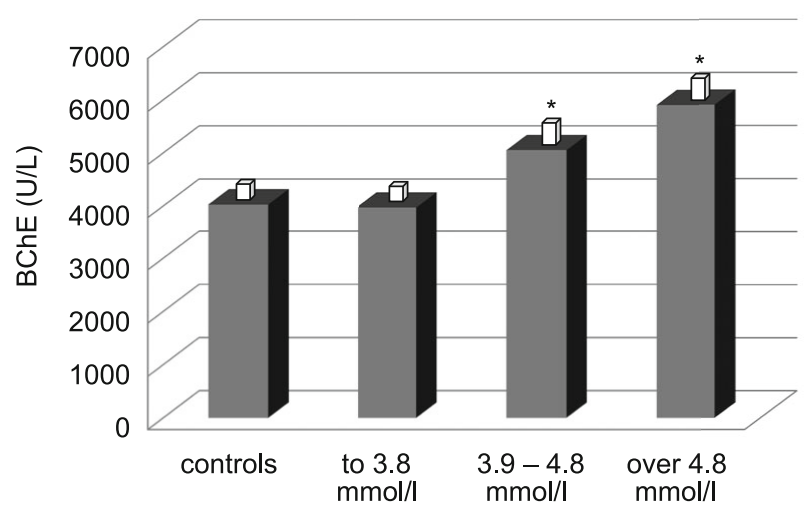

Fig. 3. BChE activity in the serum of patients with NAFLD divided by the level of non-HDL-cholesterol. *Statistically significantly higher than controls and patients with non-HDL-cholesterol less than 3.8 mmol. $\mathbf{l}^{-1}$. range $\left(56 \mathrm{ng} \cdot \mathrm{ml}^{-1}\right)$. In the group of patients with NAFLD, there were only 7 patients with hyaluronic acid values higher than the upper limit of the reference range. The serum concentration of prealbumin, which we examined as an indicator of hepatic proteosynthetic function, was practically the same in NAFLD patients as in the control group. After dividing NAFLD patients according to hyaluronic acid values into the group up to $56 \mathrm{ng} \cdot \mathrm{ml}^{-1}$ and above $56 \mathrm{ng} \cdot \mathrm{ml}^{-1}$, the prealbumin concentration in the group with increased hyaluronic acid levels was significantly lower than in the group with normal hyaluronic acid levels $\left(140 \pm 12 \mathrm{mg} . \mathrm{l}^{-1} \mathrm{vs}\right.$ $\left.250 \pm 8 \mathrm{mg} \cdot \mathrm{l}^{-1}\right)$. There was a significant correlation between prealbumin and hyaluronic acid levels (correlation coefficient: $0.49, \mathrm{p}$ $<05$ ) in our group of NAFLD patients (Fig. 1).

As we were interested in the relationship of increased butyrylcholinesterase in relation to the severity of liver damage (assessment of liver proteosynthetic function and liver fibrogenesis) as well as the relationship of butyrylcholinesterase activity to lipoprotein metabolism, we evaluated butyrylcholinesterase activity in relation to prealbumin, triacylglycerol, non-HDL-cholesterol levels and markers of fibrogenesis.

We were interested in how BChE activity in NAFLD patients was related to lipoprotein metabolism. The most significant changes in lipid parameters were observed for triacylglycerols, which increased by about $90 \%$ compared to the controls. We divided NAFLD patients into the group with a TAG concentration up to 1.7 mmol..$^{-1}$ and the group with a TAG concentration higher than 1.7 mmol..$^{-1}$. As our results showed, in the group of patients with serum TAG concentration higher than $1.7 \mathrm{mmol}^{-\mathrm{l}^{-1}}$, BChE activity was significantly higher than in the group with TAG up to 1.7 mmol..$^{-1}\left(5204 \pm 291\right.$ U. ${ }^{-1}$ vs $4304 \pm 187$ U. $^{l^{-1}}$, p < 0.005) (Fig. 2) as well as in comparison with healthy controls $\left(5204 \pm 291 \mathrm{U}^{-1^{-1}} \mathrm{vs}\right.$

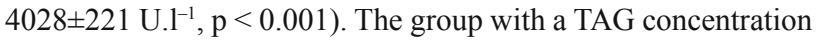
up to $1.7 \mathrm{mmol} . \mathrm{l}^{-1}$ did not differ statistically significantly from the

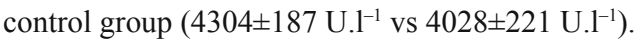

When we divided patients with NAFLD according to the level of non-HDL-cholesterol into groups up to $3.8 \mathrm{mmol} . \mathrm{l}^{-1}, 3.9-4.8$ mmol. $1^{-1}$ and above $4.8 \mathrm{mmol} . \mathrm{l}^{-1}$, it was shown that the patients with non-HDL-cholesterol above $3.8 \mathrm{mmol} . \mathrm{l}^{-1}$ had statistically significantly higher $\mathrm{BChE}$ activities than the patients with non-HDLcholesterol up to $3.8 \mathrm{mmol}^{-1}$ and healthy controls $(\mathrm{p}<0.002)$ (Fig. 3). Patients with a concentration of non-HDL-cholesterol higher than $4.8 \mathrm{mmol} . \mathrm{I}^{-1}$ had values of BChE activity statistically significantly higher than the patients with non-HDL-cholesterol in

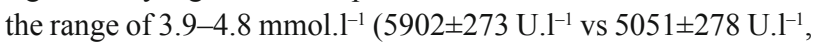
$\mathrm{p}<0.02)$. NAFLD patients, who had non-HDL-cholesterol levels below $3.8 \mathrm{mmol} . \mathrm{l}^{-1}$ had practically the same BChE activity as the

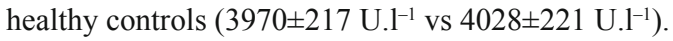

Due to the fact that the NAFLD group included both patients with simple hepatic steatosis and patients with non-alcoholic steatohepatitis (NASH), we examined in the patients with NAFLD prealbumin levels, a marker of hepatic proteosynthetic function, and hyaluronic acid, which is accepted as fibro marker. We also used the FIB-4 index to assess the liver fibrogenesis activity of patients with NAFLD. Patients with prealbumin levels higher than $200 \mathrm{mg} \cdot \mathrm{l}^{-1}$ had significantly higher BChE activity than the healthy 
BChE activity according to prealbumin

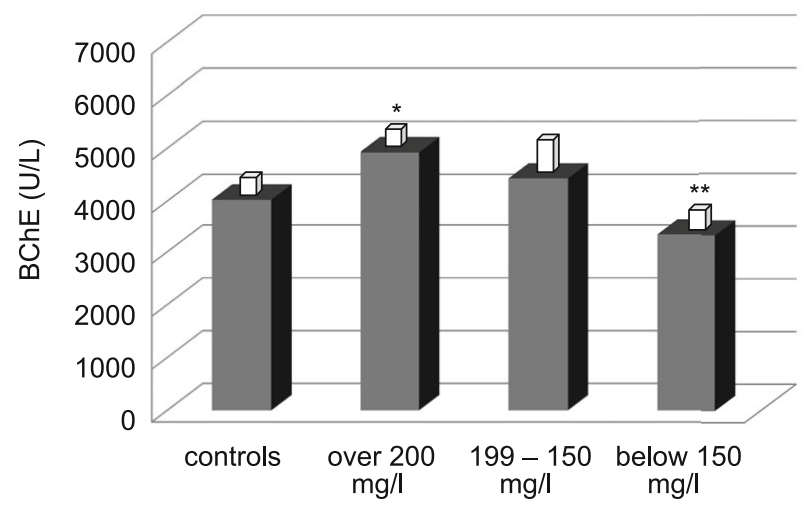

Fig. 4. BChE activity in the serum of patients with NAFLD divided by the level of prealbumin. *Statistically significantly higher than in controls $(\mathbf{p}<0.05) . * *$ Statistically significantly lower than in controls $(\mathbf{p}<\mathbf{0 . 0 5})$.

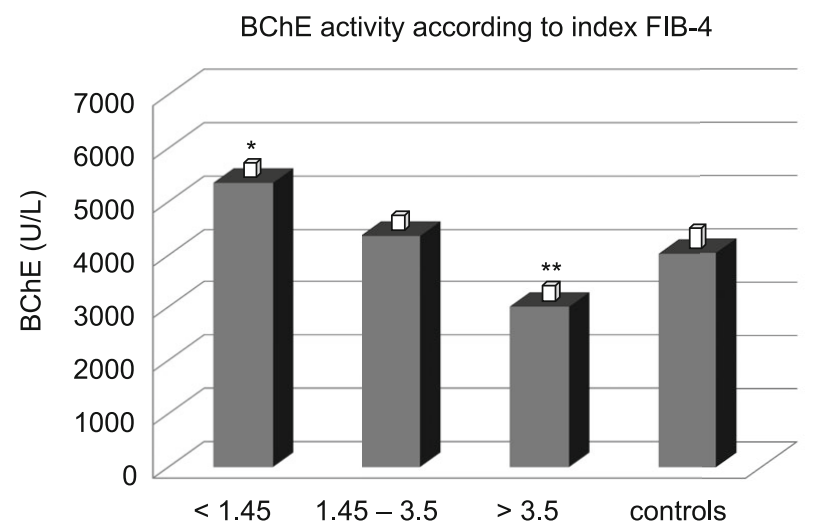

Fig. 5. BChE activity in the serum of patients with NAFLD divided by the FIB-4 index. *Statistically significantly higher than in controls (p $<0.001)$. ${ }^{* *}$ Statistically significantly lower than in controls $(\mathbf{p}<0.01)$.

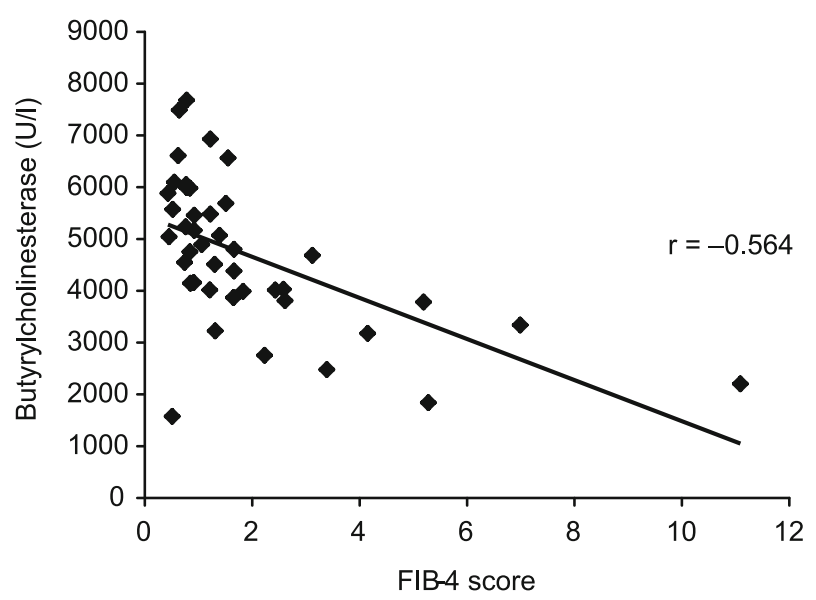

Fig. 6. Correlation between BChE activity and FIB-4 index in patients with NAFLD $(p<0.005)$.

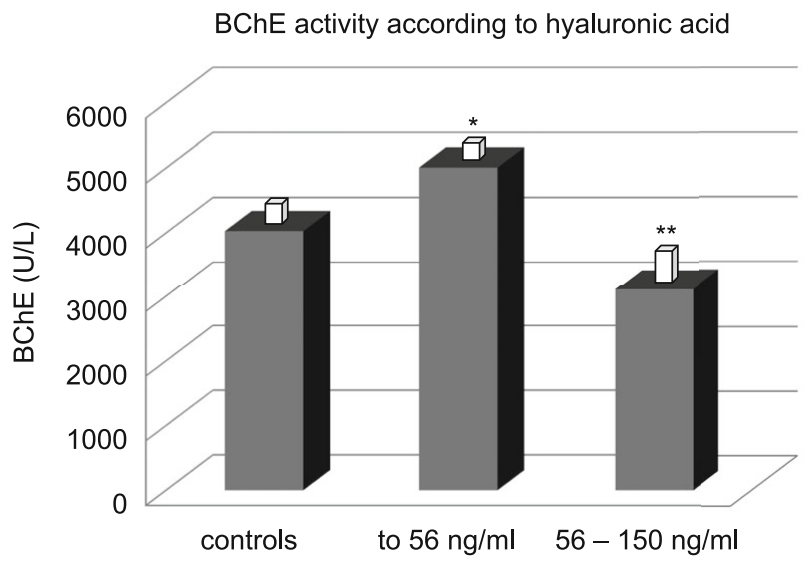

Fig. 7. BChE activity in the serum of patients with NAFLD divided by the serum hyaluronic acid level. *Statistically significantly higher than in controls $(p<0.005) . *$ Statistically significantly lower than in controls $(p<0.01)$.

controls (4923 \pm 227 U.1 $^{-1}$ vs $4028 \pm 221$ U. $1^{-1}$, p < 0.02) (Fig. 4). Patients with prealbumin concentrations of $199-150 \mathrm{mg} . \mathrm{l}^{-1}$ had higher $\mathrm{BChE}$ activity than the healthy controls, but this difference was not statistically significant (4430 $\pm 317 \mathrm{U} .1^{-1}$ vs $\left.4028 \pm 221 \mathrm{U} .1^{-1}\right)$. Patients with serum prealbumin concentrations below $150 \mathrm{mg} . \mathrm{l}^{-1}$ had significantly lower BChE activities than in the control group

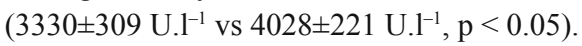

In the next part, we analysed the dependence of changes in $\mathrm{BChE}$ activity on fibrogenesis activity. To assess the activity of fibrogenesis in the liver, we used both the FIB-4 index and the level of hyaluronic acid, accepted as a fibro marker. We divided the patients with NAFLD according to the FIB-4 value into three groups-patients with FIB-4 less than 1.45, with FIB-4 in the range of 1.45-3.25 and patients with FIB-4 higher than 3.25. The results of the BChE activity showed (Fig. 5) that the highest enzyme activities were in the group with a FIB-4 values $<1.45$, i.e. in the group with F0-F1 fibrosis according to Ishak and the lowest in the group with FIB-4 higher than 3.25, i.e. in the F4-F6 fibrosis group. Patients with FIB-4 less than 1.45 had a higher BChE activity than the control group (5352 \pm 207 U. $1^{-1}$ vs $4028 \pm 221$ U. $\left.^{-1}, p<0.001\right)$. Patients with FIB-4 in the range of 1.45-3.25 had almost the same BChE activity as the

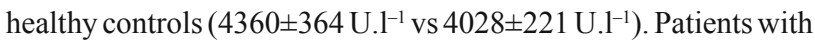
FIB-4 higher than 3.25 , i.e. patients with more severe fibrosis, had BChE activities significantly lower than the controls $(3002 \pm 268$ U. $1^{-1}$ vs $4028 \pm 221$ U. $\left.1^{-1}, p<0.01\right)$. The difference in BChE activity between the group with FIB-4 less than 1.45 and the group with FIB-4 in the range of 1.45-3.25 was statistically significant

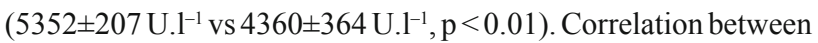
FIB-4 score and butyrylcholinesterase activities was statistically significant (correlation coefficient $=-0.564, \mathrm{p}<0.005$ ) (Fig. 6).

We also evaluated fibrogenesis activity based on the examination of serum hyaluronic acid concentration. We divided the patients with NAFLD into two groups according to their serum hyaluronic acid concentration: patients with the concentration < $56 \mathrm{ng} . \mathrm{l}^{-1}$ and patients with the concentration of 56-150 ng. $\mathrm{l}^{-1}$. In our group of patients with NAFLD, there was not a single patient 
with a hyaluronic acid concentration $>150$ ng. $\mathrm{l}^{-1}$. Patients with hyaluronic acid concentration less than $56 \mathrm{ng} \cdot 1^{-1}$ had a statistically significantly higher $\mathrm{BChE}$ activity than those in the control group $\left(5007 \pm 173\right.$ U..$^{-1}$ vs $4028 \pm 221$ U. $\left..1^{-1}, p<0,005\right)$ (Fig. 7). The patients with hyaluronic acid concentrations in the range of $56-150$ ng. $1^{-1}$ had a significantly lower BChE activity than the controls

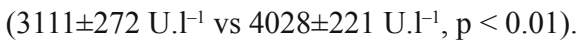

\section{Discussion}

Non-alcoholic liver disease (NAFLD) is the most common cause of chronic liver disease in Western countries that is predicted to become also the most frequent indication for liver transplantation by 2030 (14). NAFLD is a term that encompasses a broad spectrum ranging from simple steatosis to non-alcoholic steatohepatitis (NASH). It is characterized by an excess accumulation of triacylglycerols within the liver (defined as $>5 \%$ ). This disorder affects approximately $20 \%$ of the general population and up to $70 \%$ of patients with type II diabetes mellitus (15). Although hepatic biopsy is the gold standard to diagnose NAFLD, the procedure comes with a risk for major complications in $1 \%$ to $3 \%$ of the patients (16). Therefore, there is currently an effort to use non-invasive methods such as abdominal ultrasonography, magnetic resonance spectroscopy, magnetic resonance imaging, transient elastography and laboratory methods.

Cholinesterase is a secretory enzyme synthesized in the liver and secreted into the blood. The exact physiological function of $\mathrm{BChE}$ is not yet fully understood. At present, the main indications for examination of $\mathrm{BChE}$ activity in serum are assessment of liver proteosynthetic function and suspicion of organophosphate intoxication. Thomas (17) reports that serum cholinesterase activity may be increased in the patients with hepatic steatosis, diabetes mellitus and patients with hyperlipoproteinaemia type IV. Ramirez-Jimenez et al (18), Han et al (19) and Valle-Martos et al (20) described a positive association of an increased plasma $\mathrm{BChE}$ activities with triacylglycerols, total cholesterol, LDL-cholesterol, lower HDLcholesterol and metabolic syndrome. The results of the examination of BChE activity in our group of patients with NAFLD showed that the activity of BChE was significantly increased. Recently, increased cholinesterase activities in the patients with NAFLD was described by Urata et al (21).

We were interested if this increase was related to a change in lipoprotein metabolism in these patients. The most significant change in lipid parameters was observed, when examining the concentration of triacylglycerols in the serum. Serum triacylglycerol levels were practically doubled in NAFLD patients compared to the healthy controls. After dividing patients with NAFLD into the group with triacylglycerols less than $1.7 \mathrm{mmol}^{1-1}$ (reference values) and with triacylglycerols higher than $1.7 \mathrm{mmol} . \mathrm{l}^{-1}, \mathrm{BChE}$ activity was shown to be in the group with triacylglycerols higher than $1.7 \mathrm{mmol} .1^{-1}$ significantly increased compared to the controls and group of patients with TAG levels below $1.7 \mathrm{mmol}^{-\mathrm{l}^{-1}}$. Triacylglycerols are present in serum mainly in the form of VLDL, IDL and chylomicrons. This group of lipoproteins is relatively well characterized by the value of non-HDL cholesterol. The role of altered metabolism of triacylglycerol-containing lipoproteins in increasing $\mathrm{BChE}$ activity is also supported by the findings of increased BChE activity in the patients with elevated non-HDLcholesterol levels. Patients with hepatic steatosis and physiological levels of non-HDL cholesterol had practically the same BChE activities as the healthy controls. The results of our study also showed a very good correlation between BChE activity and nonHDL cholesterol concentration. Hypertriacylglycerolemia in the patients with NAFLD can be caused by an increased synthesis and/ or defective clearance of TAG-rich lipoproteins. The results of the study by Rustemeijer et al, who studied the relationship between triacylglycerol metabolism and $\mathrm{BChE}$ activity in the patients with type II diabetes, suggest that an increase in $\mathrm{BChE}$ activity was associated with an increase in VLDL-TAG synthesis.

As mentioned above, the diagnosis of NAFLD includes both patients with benign simple hepatic steatosis and patients with more severe steatohepatitis (NASH), who have a much worse prognosis. Since NASH may progress to cirrhosis, it is important to differentiate NASH from simple steatosis in its early stages. We were interested in whether there were differences in $\mathrm{BChE}$ activity between different groups of the patients with NAFLD and whether these differences could be used to detect the transition of simple hepatic steatosis to NASH.

The transition of simple liver steatosis to NASH is accompanied by several morphological (inflammation, ballooning and fibrosis) as well as functional changes. Of note, among NAFLD histologic features, the degree of liver fibrosis is the most closely associated with liver-related mortality (22). To evaluate fibrogenesis activity, we used serum hyaluronic acid concentration and FIB-4 score. Hyaluronic acid is a polysacharide synthesized by hyaluronic synthases on the plasma membrane of hepatic stellate cells, is excreted by the liver and its serum concentrations have been shown to correlate with fibrosis stage $(23,24)$. Several papers describe the use of FIB-4 index testing to assess liver fibrosis in the patients with chronic hepatopathies $(25,26)$. Kobayashi et al (27) reported that FIB-4 index could differentiate between NASH and NAFLD. We evaluated the liver functional state based on examination of the level of prealbumin in serum, which was considered to be a good indicator of proteosynthetic function of the liver (28).

Comparison of both parameters evaluating fibrogenesis in the liver with $\mathrm{BChE}$ activity showed that the $\mathrm{BChE}$ value correlated well with both the hyaluronic acid level and the FIB-4 score. NAFLD patients with HA values in the reference range had BChE activities significantly higher than the controls, while the patients with elevated $\mathrm{HA}$ values had $\mathrm{BChE}$ activities significantly lower than the healthy controls. Similarly, in patients with elevated FIB-4 scores, $\mathrm{BChE}$ activities were significantly lower than in the patients with low FIB-4 values. Comparison of BChE activity with serum prealbumin level showed a higher $\mathrm{BChE}$ activity in patients with a good liver function than in the control group, while patients with a reduced hepatic proteosynthetic function had lower BChE activity than the healthy controls. These results suggest that examination of BChE activity in the patients with NAFLD could help distinguish the patients with simple hepatic steatosis from the patients with a more advanced disease. 
689-694

\section{Conclusions}

Our results showed increased serum BChE activities in the patients with NAFLD. BChE values correlated with the severity of the lipid disorder assessed by the examination of serum TAG and non-HDL cholesterol levels as well as fatty liver index values. The comparison of changes in $\mathrm{BChE}$ activity with the changes in prealbumin levels and both markers of fibrogenesis (hyaluronic acid and FIB-4 score) showed that examination of BChE activity could help to differentiate NAFLD patients with simple hepatic steatosis from those with advanced disease such as steatohepatitis. Patients with a simple steatosis (normal proteosynthetic function, minimal changes of fibromarkers) had a significantly increased activity of BChE. NAFLD patients with more advanced disease (decreased proteosynthetic function, increased levels of fibromarkers) had a significantly decreased serum BChE activity.

\section{References}

1. Wierzbicki AS, Oben J. Nonalcoholic fatty liver and lipids. Curr Opinion Lipidol 2012; 23 (4): 345-352.

2. Maratos-Flier E. Fatty liver and FGF21 physiology. Experimental Cell Res 2017; 360 (1): 2-5.

3. Chitturi S, Farrell GC, Hashimoto E, Saibara T, Lau GK, Sollano JD. Non-alcoholic fatty liver disease in the Asia-Pacific region: definitions and overview of proposal guidelines. J Gastroenterol Hepatol 2007; 22 (6): 778-787.

4. Fan JG, Farrell GC. Epidemiology of non-alcoholic fatty liver disease in China. J Hepatol 2009; 50 (1): 204-210.

5. Platteborze PL. Acetylcholinesterase and butyrylcholinesterase. In: Kazmierczak SC, Azzazy HME (eds): Diagnostic enzymology. Berlin/ Boston: Walter de Gruyter GmbH, 2014, 197s.

6. Müller TC, Rocha JB, Morsch VM, Neis RT, Schetinger MP. Antidepressants inhibit human acetylcholinesterase and butyrylcholinesterase activity. Biochim Biophys Acta 2002; 1587 (1): 92-98.

7. Lunkes GI, Stefanello F, Lunkes DS, Morsch VM, Schetinger MRC, Goncalves JF. Serum cholinesterase activity in diabetes and associated pathologies. Diabetes Res Clin Pract 2006; 72: 28-32.

8. Rustemeijer C, Schouten JA, Voerman HJ, Beynen AC, Donker AJM, Heine RJ. Is pseudocholinesterase activity related to markers of triacylglycerol synthesis in type II diabetes mellitus? Clin Science 2001; 101: 29-35.

9. Turecký L, Kupčová V, Mojto V, Smutný M, Uhlíková E, Vozár I. Serum cholinesterase activity and proteosynthetic function of liver in patients with diabetes mellitus. Bratisl Med J 2005; 106 (8-9): 266-269.

10. Bedogni G, Bellentani S, Miglioli L, Masutti F, Passalacqua M, Castiglione A, Tiribelli $\mathbf{C}$. The fatty liver index: a simple and accurate predictor of hepatic steatosis in the general population. BMC Gastroenterol 2006; 6: 33. DOI: 10.1186/1471-230X-6-33.

11. Fedchuk L, Nascimbeni F, Pais R, Charlotte F, Housset C, Ratziu V. Performance and limitations of steatosis biomarkers in patients with nonalcokolic fatty liver disease. Aliment Pharmacol Therap 2014; 40 (10): $1209-1222$.

12. Motamed N, Sohrabi M, Ajdarkosh M et al. Fatty liver index vs waist circumference for predicting non-alcoholic fatty liver disease. World J Gastroenterol 2016; 22 (10): 3023-3030.
13. Vallet-Pichard A, Mallet V, Nalpas B et al. FIB-4: an inexpensive and accurate marker of fibrosis in $\mathrm{HCV}$ infection. Comparison with liver biopsy and FibroTest. Hepatology 2007; 46 (1): 32-36.

14. Byrne CD, Targher G. NAFLD: A multisystem disease. J Hepatol 2015; 62: 547-564.

15. Chalasani N, Younossi Z, Lavine JE et al. The diagnosis and management of nonalcoholic fatty liver disease: practice guideline by the American Association for the Study of Liver Diseases, American College of Gastroenterology, and the American Gastroenterological Association. Hepatology 2012; 55 (6): 2005-2023.

16. Bravo AA, Sheth SG, Chopra S. Liver biopsy. N Engl J Med 2001; 344 (7): 495-500.

17. Thomas L. Cholinesterasen. In: L. Thomas (ed): Labor und Diagnose. Frankfurt/Main: TH-Books Verlagsgesellschaft mbH, 2012, 98-105.

18. Ramirez-Jimenez R, Martinez-Salazar MF, Almenares-Lopez D, Yanez-Estrada L, Monroy-Noyola A. Relationship between paraoxonase-1 and butyrylcholinesterase activities and nutritional status in Mexican children. Metab Syndr Relat Disord 2018; 16 (2): 90-96.

19. HanY, Ma Y, Liu Y et al. Plasma cholinesterase is associated with Chinese adolescent overweight or obesity and metabolic syndrome prediction. Diabetes metab Syndr Obes 2019; 12: 685-702.

20. Valle-Martos R, Valle M, Martos R et al. Liver enzymes correlate with metabolic syndrome, inflammation and endothelial dysfunction in prepubertal children with obesity. Front Pediat 2021. DOI: 10.3389/ fped.2021.629346.

21. Urata N, Kawanaka M, Nishino K et al. Fatty liver and not visceral fat is more associated with liver fibrosis and diabetes in non-obese Japanese individuals: A cross-sectional study. Life (Basel) 2020; 10: 175. DOI: 10.3390/life10090175.

22. Dulai PS, Singh S, Patel J et al. Increased risk of mortality by fibrosis stage in nonalcoholic fatty liver disease: systematic review and metaanalysis. Hepatology 2017; 65 (5): 1557-1565.

23. Patel PJ, Connoley D, Rhodes F, Srivastava A, Rosenberg W. A review of the clinical utility of the Enhanced Liver Fibrosis test in multiple aetiologies of chronic liver disease. Ann Clin Biochem 2020; 57 (1): 36-43.

24. Prystupa A, Kicinski P, Luchowska-Kocot D et al. Analysis of novel serum markers of fibrosis and angiogenesis in patients with alcoholic liver cirrhosis. Ann Agric Environm Med 2020; 27 (4): 568-573.

25. Schreiner AD, Zhang J, Durkalski-Mauldin V et al. Advanced liver fibrosis and the metabolic syndrome in a primary care setting. Diabetes Metab Res Rev 2021. DOI: 10.1002/dmrr.3452.

26. Mendoza YP, Shengir M, Bosch J, Sebastiani G, Berzigotti A. FIB-4 improves LSM-based prediction of complications in overweight or obese patients with compensated advanced chronic liver disease. Clin Gastroenterol Hepatol 2021. DOI: 10.1016/j.cgh.2021.03.007.

27. Kobayashi N, Kumada T, Toyoda $\mathbf{H}$ et al. Ability of cytokeratin-18 and FIB-4 index to diagnose overall and mild fibrosis nonalcoholic steatohepatitis in Japanese nonalcoholic fatty liver disease patients. Dig Dis 2017; 35 (6): 521-530.

28. Gressner AM, Arndt T. Lexikon der Medizinischen Laboratoriumsdiagnostik. Berlin-Heidelberg: Springer Verlag 2013, 1451 pp.

Received March 16, 2021. Accepted April 24, 2021. 